\title{
Symmetric Informationally Complete Quantum Measurements
}

\author{
Joseph M. Renes ${ }^{1}$, Robin Blume-Kohout ${ }^{2}$, A. J. Scott ${ }^{1}$, and Carlton M. Caves ${ }^{1}$ \\ ${ }^{1}$ Department of Physics and Astronomy, \\ University of New Mexico, \\ Albuquerque, New Mexico 87131-1156, USA \\ ${ }^{2}$ Theoretical Division, LANL, MS-B213, \\ Los Alamos, New Mexico 87545, USA
}

(Dated: 2003 October 13)

\begin{abstract}
We consider the existence in arbitrary finite dimensions $d$ of a POVM comprised of $d^{2}$ rank-one operators all of whose operator inner products are equal. Such a set is called a "symmetric, informationally complete" POVM (SIC-POVM) and is equivalent to a set of $d^{2}$ equiangular lines in $\mathbb{C}^{d}$. SIC-POVMs are relevant for quantum state tomography, quantum cryptography, and foundational issues in quantum mechanics. We construct SIC-POVMs in dimensions two, three, and four. We further conjecture that a particular kind of group-covariant SIC-POVM exists in arbitrary dimensions, providing numerical results up to dimension 45 to bolster this claim.
\end{abstract}

\section{INTRODUCTION}

In quantum theory, measurements are represented by positive operator valued measures (POVMs). A POVM is termed informationally complete if its statistics determine completely the quantum state on which the measurement is carried out $[1-3,16]$. In order to be maximally efficient at determining the state, such a measurement should also be rank-one; i.e., the measurement operators or POVM elements should be positive multiples of projectors onto pure states, in which case each POVM element corresponds uniquely (up to a phase) to a subnormalized vector in $\mathbb{C}^{d}$. A particularly appealing and potentially useful measurement is one which is symmetric, meaning all pairwise inner products between the POVM elements are equal. Such a POVM is a "symmetric, informationally complete positive operator-valued measure," or SIC-POVM for short. The set of vectors comprising a SIC-POVM has also been studied in a very different context, where it has a different name: it is a set of $d^{2}$ equiangular lines in $\mathbb{C}^{d}$, first studied by Lemmens and Seidel [4] and subsequently by many others [5-12]. In quantum information theory such measurements are relevant to quantum state tomography [13], quantum cryptography [14], and to foundational studies [15] where they would make for a particularly interesting "standard quantum measurement". The outstanding question we address in this paper is whether SIC-POVMs exist in any finite dimension.

We conjecture that SIC-POVMs exist in all finite dimensions and, moreover, that there exists in all finite dimensions a SIC-POVM that is covariant under a standard representation of $\mathbb{Z}_{d} \times \mathbb{Z}_{d}$. To state the conjecture, let us formalize the definition of a SIC-POVM. The simplest definition is that a SIC-POVM $P$ is a set of $d^{2}$ normalized vectors $\left|\phi_{k}\right\rangle$ in $\mathbb{C}^{d}$ satisfying

$$
\left|\left\langle\phi_{j} \mid \phi_{k}\right\rangle\right|^{2}=\frac{1}{d+1}, \quad j \neq k .
$$

More precisely, the POVM elements of $P$ are the subnormalized projectors $\left|\phi_{k}\right\rangle\left\langle\phi_{k}\right| / d=\Pi_{k} / d$, which have pairwise Hilbert-Schmidt inner product $\left(\Pi_{j}, \Pi_{k}\right) / d^{2}=\operatorname{Tr}\left[\Pi_{j}^{\dagger} \Pi_{k}\right] / d^{2}=1 / d^{2}(d+1)$ for $j \neq k$. It turns out that the other properties of a SIC-POVM, i.e., completeness and informational completeness, follow from Eq. (1), as we show in section 2 .

We can now state our conjecture.

Conjecture 1 For any dimension $d \in \mathbb{N}$, let $\{|k\rangle\}_{k=0}^{d-1}$ be an orthonormal basis for $\mathbb{C}^{d}$, and define

$$
\omega=\exp (2 \pi i / d), \quad D_{j k}=\omega^{j k / 2} \sum_{m=0}^{d-1} \omega^{j m}|k \oplus m\rangle\langle m|
$$

where $\oplus$ denotes addition modulo $d$. Then there exists a normalized $|\phi\rangle \in \mathbb{C}^{d}$ such that the set $\left\{D_{j k}|\phi\rangle\right\}_{j, k=1}^{d}$ is a SIC-POVM P.

Analytic solutions are known for $d=2,3,8$ [8], and to this list we add $d=4$. Additionally, computer calculations reveal numerical solutions (with an accuracy better than 1 part in $10^{8}$ ) in dimensions up to 45 , some derived using the aforementioned group, but others using other suitable groups. These results are detailed herein according to the 
following plan. Section 2 states the problem in the language of frame theory and derives a connection to the problem of finding spherical $t$-designs. Section 3 specializes to the group-covariant case and explains why our conjecture might be generally true. Section 4 presents our analytic solutions for $d=2,3,4$, and section 5 the method of obtaining the numerical results. Finally, in section 6 we discuss possible approaches to a general proof, as well as related open questions.

\section{FRAMES AND SPHERICAL $t$-DESIGNS}

The concepts of frame theory provide a simple and elegant means of putting our problem in a general setting, for a SIC-POVM is a particular kind of frame. Frames are a generalization of basis sets, with the requirements of orthogonality and normalization relaxed. For a finite-dimensional vector space $\mathcal{H}$, a collection of vectors $\left|\psi_{k}\right\rangle \in \mathcal{H}$ is a frame if there exist constants $0<a \leq b<\infty$ such that

$$
a|\langle\xi \mid \xi\rangle|^{2} \leq \sum_{k}\left|\left\langle\xi \mid \psi_{k}\right\rangle\right|^{2} \leq b|\langle\xi \mid \xi\rangle|^{2}
$$

for all $|\xi\rangle \in \mathcal{H}$. Any collection of vectors is a frame in the subspace spanned by the vectors. The constants $a$ and $b$ are called the frame bounds, and if $a=b$, the frame is said to be tight. The frame operator is the positive operator

$$
S=\sum_{k}\left|\psi_{k}\right\rangle\left\langle\psi_{k}\right|
$$

It should be immediately clear that for a tight frame $S=a I$. This tight-frame condition is equivalent to the completeness condition for the corresponding POVM elements $\left|\psi_{k}\right\rangle\left\langle\psi_{k}\right| / a$, and thus rank-one POVMs and tight frames are the same mathematical object.

Now let $\mathbb{S}^{d} \subset \mathbb{C}^{d}$ be the subset consisting of vectors that have unit norm. Any frame can be rewritten in terms of the corresponding normalized vectors, but tightness is not preserved under this transformation. For a frame $\left\{\left|\psi_{k}\right\rangle \in \mathbb{S}^{d}\right\}_{k=1}^{n}$ made up of normalized vectors, the quantity

$$
\operatorname{Tr}\left[S^{2}\right]=\sum_{j, k}\left|\left\langle\psi_{j} \mid \psi_{k}\right\rangle\right|^{2}
$$

is called the frame potential. We consider only frames made up of normalized vectors throughout the following.

A useful theorem due to Benedetto and Fickus [18] states the following.

Theorem 1 (Benedetto-Fickus) Given any $d$ and $n$, let $\left\{\left|\psi_{k}\right\rangle \in \mathbb{S}^{d}\right\}_{k=1}^{n}$ be a set of normalized vectors with frame operator $S$. Then

$$
\operatorname{Tr}\left[S^{2}\right] \geq \max \left(n, n^{2} / d\right)
$$

Furthermore, the bound is achieved if and only if $\left\{\left|\psi_{k}\right\rangle\right\}$ consists of orthonormal vectors, when $n \leq d$, or is a tight frame, when $n \geq d$.

Proof The proof is so simple that we can include it here for completeness. Denoting the ordered eigenvalues of $S$ by $\lambda_{1} \geq \lambda_{2} \geq \ldots \geq \lambda_{d}$, we first note that the number of nonzero eigenvalues is at most $q=\min (n, d)$. Thus we have

$$
\operatorname{Tr}[S]=n=\sum_{k=1}^{q} \lambda_{k} \quad \text { and } \quad \operatorname{Tr}\left[S^{2}\right]=\sum_{k=1}^{q} \lambda_{k}^{2} .
$$

Minimizing $\operatorname{Tr}\left[S^{2}\right]$ subject to the constraint $\operatorname{Tr}[S]=n$ gives the inequality. Equality holds if and only if $\lambda_{k}=n / q$, $k=1, \ldots, q$. Thus for $n \leq d, S$ is a projector onto an $n$-dimensional subspace, implying that the vectors $\left|\psi_{k}\right\rangle$ are orthogonal, and if $n \geq d, S=(n / d) I$, implying that the set $\left\{\left|\psi_{k}\right\rangle\right\}$ is a tight frame.

Since the frame potential of $P$ is $\operatorname{Tr}\left[S^{2}\right]=d^{3}=n^{2} / d$, the theorem establishes immediately that $P$ is a tight frame, whence $P$ is also a POVM.

For $P$ to be informationally complete, the $d^{2}$ operators $\Pi_{k}=\left|\phi_{k}\right\rangle\left\langle\phi_{k}\right|$ must be linearly independent so that they span the space of operators. The linear independence follows from considering the rank of their Gram matrix $\left(\Pi_{j}, \Pi_{k}\right)=\operatorname{Tr}\left[\Pi_{j}^{\dagger} \Pi_{k}\right]=\left(d \delta_{j k}+1\right) /(d+1)$, which being circulant (each row is a cyclic shift of the previous row), has eigenvalues given by the Fourier transform of one of the rows. A simple calculation reveals that due to the combination of constant term and Kronecker delta, the eigenvalues are exactly the same as the values in any row. 
Since no eigenvalues are zero, the Gram matrix has full rank, the projection operators $\Pi_{k}$ are linearly independent, and $P$ is informationally complete.

Since every rank-one POVM is a tight frame, a SIC-POVM $P$ is clearly something more. To fully elucidate the properties and applications of $P$, we need to introduce spherical $t$-designs. Building on the result of Benedetto and Fickus, we can establish a connection between frames and spherical $t$-designs applicable to the SIC-POVM problem.

A spherical $t$-design is a set of $n$ normalized vectors $\left\{\left|\phi_{k}\right\rangle \in \mathbb{S}^{d}\right\}$ such that the average value of any $t$-th order polynomial $f_{t}(\psi)$ over the set $\left\{\left|\phi_{k}\right\rangle\right\}$ is equal to the average of $f_{t}(\psi)$ over all normalized vectors $|\psi\rangle$. Note that if a set is a $t$-design, it is also an $s$-design for all $s \leq t$, since an $s$-th order polynomial is also a $t$-th order polynomial. Spherical $t$-designs were originally developed as subsets of the real sphere $S^{d}$; here we apply the concept to the set $\mathbb{S}^{d}$.

Let $\mathcal{H}=\mathbb{C}^{d}, \mathcal{H}_{t}$ be the $t$-fold tensor product of such spaces, and $\mathcal{S}_{t}$ be the symmetric subspace of $\mathcal{H}_{t}$, and consider a function $f_{t}: \mathcal{H} \rightarrow \mathbb{C}$ defined as

$$
f_{t}(\psi)=\left\langle\Psi^{t}\left|F_{t}\right| \Psi^{t}\right\rangle, \quad\left|\Psi^{t}\right\rangle=|\psi\rangle^{\otimes t}, \quad|\psi\rangle \in \mathcal{H},
$$

where the choice of $f_{t}$ is equivalent to a choice of a symmetric operator $F_{t} \in \mathcal{B}\left(\mathcal{S}_{t}\right)$. Such a function is a $t$-th order polynomial function on $\mathcal{H}$. We can decompose $F_{t}$ into a sum of product operators, i.e., $F_{t}=\sum_{k} \bigotimes_{j=1}^{t} A_{j ; k} ;$ thus any such function can be decomposed into monomial terms like

$$
\left\langle\Psi^{t}\left|\bigotimes_{j=1}^{t} A_{j}\right| \Psi^{t}\right\rangle=\prod_{j=1}^{t}\left\langle\psi\left|A_{j}\right| \psi\right\rangle
$$

Without loss of generality, we can restrict our attention to such monomial functions and rewrite them as

$$
f_{t}(\psi)=\prod_{j=1}^{t} \operatorname{Tr}\left[A_{j}|\psi\rangle\langle\psi|\right]=\operatorname{Tr}\left[\left(\bigotimes_{j=1}^{t} A_{j}\right) \Pi_{\psi}^{\otimes t}\right], \quad \Pi_{\psi}=|\psi\rangle\langle\psi| .
$$

Since the set $\left\{\left|\phi_{k}\right\rangle\right\}$ is a $t$-design if and only if the average of any $f_{t}$ over $\left\{\left|\phi_{k}\right\rangle\right\}$ is equal to its average over all $|\psi\rangle \in \mathbb{S}^{d}$, we are led to compute the average of an arbitrary monomial term:

$$
\left\langle f_{t}\right\rangle=\int \mathrm{d} \psi \operatorname{Tr}\left[\left(\bigotimes_{j=1}^{t} A_{j}\right) \Pi_{\psi}^{\otimes t}\right]=\operatorname{Tr}\left[\left(\bigotimes_{j=1}^{t} A_{j}\right) \int \mathrm{d} \psi \Pi_{\psi}^{\otimes t}\right]=\operatorname{Tr}\left[\left(\bigotimes_{j=1}^{t} A_{j}\right) K_{t}\right] .
$$

Hence we focus on finding $K_{t}$, since it effectively takes the average of $f_{t}$. A spherical $t$-design is then a set of vectors for which

$$
S_{t}=\sum_{k=1}^{n}\left|\Phi_{k}^{t}\right\rangle\left\langle\Phi_{k}^{t}\left|=n K_{t}, \quad\right| \Phi_{k}^{t}\right\rangle=\left|\phi_{k}\right\rangle^{\otimes t}
$$

Note that $S_{t}$ is the $t$-fold tensor-product analog of the frame operator $S$.

To find the operator $K_{t}$, note that $K_{t}$ has support only on the symmetric subspace $\mathcal{S}_{t}$. Further, because $K_{t}$ is invariant under any $U^{\otimes t}$ for $U \in S U(d)$, we conclude that $K_{t} \propto \Pi_{\text {sym }}$, the projector onto $\mathcal{S}_{t}$. (Recall that $\mathcal{S}_{t}$ is an irreducible invariant subspace of the group consisting of the operators $U^{\otimes t}$.) Finally, to determine the constant of proportionality, we consider the average of the trivial function $f_{t}(\psi)=1$. Equation $(11)$ then becomes $\operatorname{Tr}\left[K_{t}\right]=1$, and since $\mathcal{S}_{t}$ has dimension $\left(\begin{array}{c}t+d-1 \\ d-1\end{array}\right)$, we have

$$
K_{t}=\frac{t !(d-1) !}{(t+d-1) !} \Pi_{\mathrm{sym}}
$$

For $t=1$, we see that a 1-design is a tight frame made up of normalized vectors and, hence, also a POVM made up of equally weighted rank-one projectors.

Equation (12) now says that the set $\left\{\left|\phi_{k}\right\rangle\right\}$ is a $t$-design if and only if the set $\left\{\left|\Phi_{k}^{t}\right\rangle\right\}$ is a tight frame on $\mathcal{S}_{t}$, whence we can apply Theorem 1 to obtain the following result.

Theorem 2 A set of normalized vectors $\left\{\left|\phi_{k}\right\rangle \in \mathbb{S}^{d}\right\}_{k=1}^{n}$ with $n \geq\left(\begin{array}{c}t+d-1 \\ d-1\end{array}\right)$ forms a spherical $t$-design if and only if

$$
\operatorname{Tr}\left[S_{t}^{2}\right]=\sum_{j, k}\left|\left\langle\phi_{j} \mid \phi_{k}\right\rangle\right|^{2 t}=\frac{n^{2} t !(d-1) !}{(t+d-1) !} .
$$

Furthermore, this value is the global minimum of $\operatorname{Tr}\left[S_{t}^{2}\right]$. 
This theorem links the spherical $t$-design property with the minimization of the $t$-th frame potential, $\operatorname{Tr}\left[S_{t}^{2}\right]$. Immediately we can infer that every SIC-POVM is a 2 -design since $\operatorname{Tr}\left[S_{2}^{2}\right]=\sum_{j, k}\left|\left\langle\phi_{j} \mid \phi_{k}\right\rangle\right|^{4}=2 d^{3} /(d+1)$, the required value for a 2-design. The converse is also true, namely, every 2-design with $n=d^{2}$ elements is a SIC-POVM. To show this, let $\lambda_{j k}=\left|\left\langle\phi_{j} \mid \phi_{k}\right\rangle\right|^{2}, j \neq k$, and interpret these $\lambda_{j k}$ as coordinates in $\mathbb{R}^{d^{2}\left(d^{2}-1\right)}$. Using the values of the frame potentials for a 2-design with $n=d^{2}$ elements, we can write

$$
\sum_{j \neq k} \lambda_{j k}=\operatorname{Tr}\left(S^{2}\right)-d^{2}=\frac{d^{2}\left(d^{2}-1\right)}{d+1} \quad \text { and } \quad \sum_{j \neq k} \lambda_{j k}^{2}=\operatorname{Tr}\left(S_{2}^{2}\right)-d^{2}=\frac{d^{2}\left(d^{2}-1\right)}{(d+1)^{2}} .
$$

The first equation describes a plane and the second a sphere. They intersect at the single tangent point $\lambda_{j k}=1 /(d+1)$, thus showing that all 2-designs with $d^{2}$ elements are SIC-POVMs. These considerations make clear that the crucial distinguishing property of a SIC-POVM is that it is also a 2-design. Moreover, this ensures that minimizing the second frame potential, as we do in the numerical work reported in section 4, yields vectors that do indeed form a SIC-POVM.

Furthermore, $d^{2}$ is the smallest number of elements a 2-design can have, so a SIC-POVM is a minimal 2-design (see also [8]). Theorem 2 does not provide the minimum number of states, $n_{\min }$, for a $t$-design in $d$ dimensions, but we can establish lower bounds. For the $t=2$ case, consider again the steps leading to the definition of the operator $K_{t}$. In carrying out the average of the function $f_{2}$, we could have written

$$
\left\langle f_{2}\right\rangle=\operatorname{Tr}\left[A_{2} \int \mathrm{d} \psi|\psi\rangle\left\langle\psi\left|A_{1}\right| \psi\right\rangle\langle\psi|\right]=\operatorname{Tr}\left[A_{2} \mathcal{G}\left(A_{1}\right)\right]
$$

and thus considered the superoperator $\mathcal{G}: \mathcal{B}\left(\mathbb{C}^{d}\right) \rightarrow \mathcal{B}\left(\mathbb{C}^{d}\right)$. Here $\mathcal{G}\left(U A U^{\dagger}\right)=U \mathcal{G}(A) U^{\dagger}$ for any $U \in S U(d)$, so by Schur's lemma, $\mathcal{G}$ is some linear combination of projectors onto the invariant subspaces of $U$ acting on $\mathcal{B}\left(\mathbb{C}^{d}\right)$. These invariant subspaces are (i) the $\left(d^{2}-1\right)$-dimensional subspace of traceless operators and (ii) the one-dimensional subspace spanned by the identity operator $I$. Thus we can write $\mathcal{G}=a \mathcal{I}+b \mathbf{I}$ where $\mathcal{I}(A)=A$ and $\mathbf{I}(A)=\operatorname{Tr}[A] I$ (i.e., $\mathcal{I}$ is the identity superoperator, and $\mathbf{I}$ projects onto the identity operator). To find $a$ and $b$, we first let $A_{1}=A_{2}=I$, which gives the function $f_{2}(\psi)=1$, so that Eq. (16) yields $d(a+b d)=1$. Next we consider $A_{1}=A_{2}=|\phi\rangle\langle\phi|$, for which $\left\langle f_{2}\right\rangle=\int \mathrm{d} \psi|\langle\phi \mid \psi\rangle|^{4}=a+b$. We can use Eqs. (11) and (13) to show that $\left\langle f_{2}\right\rangle=2 / d(d+1)$; combined with the previous result, this implies $a=b=1 / d(d+1)$. Therefore, $\mathcal{G}$ has no null subspace, must be rank- $d^{2}$, and cannot be constructed from less than $d^{2}$ linearly independent rank-one superoperators. Similar arguments can be applied to all spherical $t$-designs. By similar rearrangements, we can make several different types of operators $K_{t}^{\prime}$, and the rank of each serves as a lower bound on the number of vectors required to comprise a $t$-design.

\section{GROUP COVARIANCE}

Our results are obtained by considering group-covariant sets, so it is appropriate to specialize to this case. The SIC-POVM $P$ is group covariant if there exists a group $G$ with a $d$-dimensional projective unitary representation $\left\{U_{g}\right\}$ such that (i) $P$ is invariant under any $U_{g}$, i.e., for any $\left|\phi_{j}\right\rangle \in P$ and any $U_{g}, U_{g}\left|\phi_{j}\right\rangle \in P$ (up to a phase), and (ii) $\left\{U_{g}\right\}$ acts transitively on $P$, i.e., for any $\left|\phi_{j}\right\rangle,\left|\phi_{k}\right\rangle \in P$, there exists $U_{g}$ such that $U_{g}\left|\phi_{j}\right\rangle=\left|\phi_{k}\right\rangle$ (also up to a phase). Assuming group covariance simplifies the search for SIC-POVMs. We simply search for a fiducial vector such that $P=\left\{U_{g}|\phi\rangle\right\}$ is a SIC-POVM (note that the transitivity property implies that the order of $G$ must be at least $d^{2}$ ). To do this, we use groups such that $\left\{U_{g}|\phi\rangle\right\}$ is a 1-design, i.e., a POVM, for any normalized vector $|\phi\rangle$, and then we search for a particular vector $|\phi\rangle$ such that $\left|\left\langle\phi\left|U_{g}\right| \phi\right\rangle\right|^{2}=1 /(d+1)$ for all $g \neq e$. All other inner products are then guaranteed to have this value due to the group action.

We suspect the case of group covariance to be general for the following reason. Consider the map $\alpha: \mathbb{S}^{d} \rightarrow \mathcal{B}\left(\mathbb{C}^{d}\right)$ that takes a normalized vector to the corresponding projector, i.e., $\alpha\left(\left|\phi_{j}\right\rangle\right)=\left|\phi_{j}\right\rangle\left\langle\phi_{j}\right|$. Now consider the operators

$$
\sigma_{j}=\sqrt{\frac{d}{d-1}}\left(\left|\phi_{j}\right\rangle\left\langle\phi_{j}\right|-\frac{I}{d}\right) .
$$

Being both traceless and Hermitian, these operators lie in a subspace of $\mathcal{B}\left(\mathbb{C}^{d}\right)$ that is isomorphic to $\mathbb{R}^{d^{2}-1}$; indeed, since $\left(\sigma_{j}, \sigma_{j}\right)=1$, they all lie on the unit sphere in $\mathbb{R}^{d^{2}-1}$. This sphere is a generalization of the Bloch sphere for two-dimensional systems, the difference being that for $d>2$, not all operators on the sphere are images of vectors in $\mathbb{S}^{d}$ under the map $\alpha$. From the SIC-POVM condition (1), one finds immediately that

$$
\left(\sigma_{j}, \sigma_{k}\right)=-\frac{1}{d^{2}-1} \quad \forall j \neq k
$$


This is the condition for the $d^{2}$ operators $\left\{\sigma_{j}\right\}$ to form a regular simplex in $\mathbb{R}^{d^{2}-1}$, whose automorphism group is the permutation group $S_{d^{2}}$. Given this result, some group covariance seems natural. One is tempted to think that from here it is a simple matter to establish the existence of the set $P$. This is not the case, however, as working in the operator space obscures the very difficult task of determining when a given operator is the image of some element of $\mathbb{S}^{d}$ under the map $\alpha$. In the same vein, most of the elements of the permutation group cannot be represented in this framework as unitary transformations of $\mathbb{C}^{d}$; thus, while we know that any $G$ satisfying the conditions above must be a subgroup of $S_{d^{2}}$, it is not obvious which subgroups are candidates.

The outstanding choice for $G$ is the group $\mathbb{Z}_{d} \times \mathbb{Z}_{d}$, as described in the conjecture. We note in passing that using this group to find a SIC-POVM makes $P$ a Gabor or Weyl-Heisenberg frame, this being the definition of such a frame [24]; such a SIC-POVM $P$ is useful in defining finite-dimensional analogs of the familiar $P$ and $Q$ quasidistributions of infinite dimensions. The group's usefulness here stems from the fact that, for any normalized $|\psi\rangle \in \mathbb{S}^{d}$,

$$
S_{\psi}=\sum_{j k} D_{j k}|\psi\rangle\langle\psi| D_{j k}^{\dagger}=d I
$$

a fact readily checked by direct calculation.

The property in Eq. (19) of producing a 1-design for any input state is quite general. The following argument is adapted from Proposition 3 of [17]. Any set of $d^{2}$ orthogonal unitary operators $T_{j}$, thus satisfying $\operatorname{Tr}\left[T_{j}^{\dagger} T_{k}\right]=d \delta_{j k}$, is a complete set for expanding operators in $\mathcal{B}\left(\mathbb{C}^{d}\right)$; the unitary operators $\left\{D_{j k}\right\}$ are one example of operators that satisfy this orthogonality condition. It is a simple matter to turn the completeness relation into $\sum_{k} T_{k} C T_{k}^{\dagger}=d \operatorname{Tr}[C] I$ for any operator $C$. Simply consider the inner product of two arbitrary operators $A$ and $B$. The completeness relation means that

$$
(A, B)=\frac{1}{d} \sum_{k}\left(A, T_{k}\right)\left(T_{k}, B\right) .
$$

Setting $A=\left|\phi_{1}\right\rangle\left\langle\phi_{2}\right|$ and $B=\left|\psi_{1}\right\rangle\left\langle\psi_{2}\right|$ (which we can do without loss of generality because such outer products span $\left.\mathcal{B}\left(\mathbb{C}^{d}\right)\right)$, we find

$$
\left\langle\phi_{1} \mid \psi_{1}\right\rangle\left\langle\psi_{2} \mid \phi_{2}\right\rangle=\frac{1}{d} \sum_{k}\left\langle\phi_{1}\left|T_{k}\right| \phi_{2}\right\rangle\left\langle\psi_{2}\left|T_{k}^{\dagger}\right| \psi_{1}\right\rangle
$$

from which it follows that $\sum_{k} T_{k}\left|\phi_{2}\right\rangle\left\langle\psi_{2}\right| T_{k}^{\dagger}=d\left\langle\psi_{2} \mid \phi_{2}\right\rangle$, whence the result follows.

Thus the property of producing a 1-design regardless of the fiducial $\left|\phi_{0}\right\rangle$ is common to all groups of size $d^{2}$ whose representation operators are a complete, orthogonal set. Such groups were introduced by Knill in connection with quantum error-correcting codes and are called "nice error bases" or unitary error bases [22, 23]. Klappenecker and Rötteler have kindly detailed all such nice error bases up to dimension 10, so we can apply them to the problem at hand [19-21]. Only the nice error bases associated with the group $\mathbb{Z}_{d} \times \mathbb{Z}_{d}$ exist in every dimension, thus accounting for our focus on this group.

\section{ANALYTIC SIC-POVMS}

Here we concentrate specifically on using the group $\mathbb{Z}_{d} \times \mathbb{Z}_{d}$. Fixing the representation operators $D_{j k}$ of this group we can determine the set of fiducial vectors that under the group action make a SIC-POVM. From this we can determine also the number of distinct SIC-POVMs generated by our fixed representation. In three dimensions there are an uncountably infinite number of such covariant SIC-POVMs, but in two dimensions there are just two, and in four, 16. We write the fiducial state as

$$
|\phi\rangle=\sum_{k} r_{k} e^{i \theta_{k}}|k\rangle
$$

where we can, of course, immediately choose $\theta_{0}=0$.

$$
\text { A. } d=2
$$

The two solutions, represented as column vectors in the standard basis, are

$$
\left\{\frac{1}{\sqrt{6}}\left(\begin{array}{c}
\sqrt{3+\sqrt{3}} \\
e^{i \pi / 4} \sqrt{3-\sqrt{3}}
\end{array}\right), \frac{1}{\sqrt{6}}\left(\begin{array}{c}
-\sqrt{3-\sqrt{3}} \\
e^{i \pi / 4} \sqrt{3+\sqrt{3}}
\end{array}\right)\right\} .
$$


These have a simple interpretation on the Bloch sphere, where the nontrivial group operators are simply rotations by $\pi$ about the $x, y$, and $z$ axes, respectively. Then the Bloch vectors of the two fiducial states are $\pm(1,1,1) / \sqrt{3}$, and the two SIC-POVM states thus formed are regular tetrahedra, each one related to the other by inversion of the Bloch vectors.

B. $d=3$

For $r_{0}$ satisfying $1 / \sqrt{2}<r_{0}<\sqrt{2 / 3}$, define

$$
r_{ \pm}\left(r_{0}\right)=\frac{1}{2} r_{0} \pm \frac{1}{2} \sqrt{2-3 r_{0}^{2}}
$$

Hence $0<r_{-} \leq 1 / \sqrt{6} \leq r_{+}<1 / \sqrt{2}<r_{0} \leq \sqrt{2 / 3}$. The complete set of fiducial states, represented as column vectors in the standard basis, is then

$$
\begin{gathered}
\left\{\left(\begin{array}{c}
r_{0} \\
r_{+} e^{i \theta_{1}} \\
r_{-} e^{i \theta_{2}}
\end{array}\right),\left(\begin{array}{c}
\text { plus all vectors formed } \\
\text { by permuting of elements }
\end{array}\right) \mid \theta_{1}, \theta_{2} \in\left\{\frac{\pi}{3}, \pi, \frac{5 \pi}{3}\right\}, \frac{1}{\sqrt{2}}<r_{0} \leq \sqrt{\frac{2}{3}}\right\} \\
\bigcup\left\{\left(\begin{array}{c}
1 / \sqrt{2} \\
e^{i \theta_{1} / \sqrt{2}} \\
0
\end{array}\right),\left(\begin{array}{l}
\text { plus all vectors formed } \\
\text { by permuting of elements }
\end{array}\right) \mid 0 \leq \theta_{1}<2 \pi\right\} . \\
\text { C. } d=4
\end{gathered}
$$

Now let

$$
r_{0}=\frac{1-1 / \sqrt{5}}{2 \sqrt{2-\sqrt{2}}}, \quad r_{1}=(\sqrt{2}-1) r_{0}, \quad r_{ \pm}=\frac{1}{2} \sqrt{1+1 / \sqrt{5} \pm \sqrt{1 / 5+1 / \sqrt{5}}}
$$

along with

$$
a=\arccos \frac{2}{\sqrt{5+\sqrt{5}}}, \quad b=\arcsin \frac{2}{\sqrt{5}}
$$

and define the set

$$
\begin{aligned}
\Omega \equiv\{ & \left((-1)^{m}(a / 2+b / 4)+\pi(m+2 n+7 j+1) / 4, \pi(2 k+1) / 2,\right. \\
& \left.\left.(-1)^{m}(-a / 2+b / 4)+\pi(m+2 n+3 j+4 k+1) / 4\right) \mid j, k, m=0,1 \text { and } n=0, \ldots, 3\right\} .
\end{aligned}
$$

The complete set of fiducial states, represented as column vectors in the standard basis, is now

$$
\left\{\left(\begin{array}{c}
r_{0} \\
r_{+} e^{i \theta_{+}} \\
r_{1} e^{i \theta_{1}} \\
r_{-} e^{i \theta_{-}}
\end{array}\right),\left(\begin{array}{c}
r_{0} \\
r_{-} e^{i \theta_{-}} \\
r_{1} e^{i \theta_{1}} \\
r_{+} e^{i \theta_{+}}
\end{array}\right),\left(\begin{array}{c}
\text { plus all vectors formed } \\
\text { by cycling of elements }
\end{array}\right) \mid\left(\theta_{+}, \theta_{1}, \theta_{-}\right) \in \Omega\right\} .
$$

\section{NUMERICAL SIC-POVMS}

Because analytic solutions to the SIC-POVM condition (1) are so few, our conjectures are based almost entirely on numerical evidence (even the $d=4$ solution was originally inspired by close examination of numerical solutions). To find numerical instances of $P$, we simply minimize the second frame potential $\operatorname{Tr}\left[S_{2}^{2}\right]$ over sets of $d^{2}$ normalized vectors generated by a representation of $\mathbb{Z}_{d} \times \mathbb{Z}_{d}$ from a vector $|\phi\rangle$. It is also possible to vary independently the $d^{2}$ 
elements of $P$, but this is much less efficient; taking advantage of the group-covariance conjecture permits us to search a space of $O(d)$ complex parameters instead of $O\left(d^{3}\right)$ complex parameters.

The quantity that we minimize, $\sum_{j, k}\left|\left\langle\phi\left|D_{j k}\right| \phi\right\rangle\right|^{4}$, is proportional to the frame potential because of the group covariance. Since it is a quartic function of $|\phi\rangle$, we have to use numerical methods to minimize it, using either Mathematica (simpler) or $\mathrm{C}++$ (much faster). The method used is an adaptive conjugate gradient method; this has the advantage of converging with exponential rapidity to a local minimum, but the disadvantage of being insensitive to global conditions. As a result, the most time-intensive portion of the computation by far is identifying one of the global minima among the many local minima.

Once the correct minimum is located, we quickly obtain $P$ such that Eq. (1) is satisfied to an accuracy of $10^{-8}$. The sole exception to this rule is $d=3$ (where an exact analytic solution is known): in $d=3$ there exists a continuously infinite family of solutions, and this degeneracy makes numerical solution difficult. For every dimension between $d=5$ and $d=45$, however, we have found $\mathbb{Z}_{d} \times \mathbb{Z}_{d}$-covariant solutions to within machine precision [25].

Additionally, in small dimensions, one can attempt an exhaustive search for all possible $\mathbb{Z}_{d} \times \mathbb{Z}_{d}$-covariant SICPOVMs, by simply running the minimization many times with differing presumptive fiducial states, tabulating all the while the distinct SIC-POVM fiducial states found. Table 1 lists the results for the number of distinct SIC-POVMs.

\begin{tabular}{c|c}
$d$ & $\#$ (SIC-POVMs) \\
\hline 2 & 2 \\
3 & $\infty$ \\
4 & 16 \\
5 & 80 \\
6 & 96 \\
7 & 336
\end{tabular}

TABLE I: Number of SIC-POVM sets generated by a fixed representation of the group $\mathbb{Z}_{d} \times \mathbb{Z}_{d}$ in dimensions two through seven. The infinity in dimension three is uncountable.

Finally, we have tested some of the other nice error bases tabulated by Klappenecker and Rötteler. These are also easy handled, and although not all groups were tested, at least four groups were found to generate SIC-POVM sets. In the notation of the library of small groups used by GAP3, GAP4, and MAGMA, these groups are G(36,11), $\mathrm{G}(36,14), \mathrm{G}(64,8)$, and $\mathrm{G}(81,9)$. Each of these solutions has an accuracy of $10^{-15}$ in the individual vector inner products. Perhaps surprisingly, many of the tabulated groups do not seem to yield group-covariant SIC-POVMs.

\section{ODDS AND ENDS}

A rigorous proof of existence of SIC-POVMs in all finite dimensions seems tantalizingly close, yet remains somehow distant. Although the numerical evidence makes very clear the relevance of the group $\mathbb{Z}_{d} \times \mathbb{Z}_{d}$, this is not definitively established. Given the apparent importance of $\mathbb{Z}_{d} \times \mathbb{Z}_{d}$, it would seem to be just a short step to some general form for an operator whose eigenvectors could be a fiducial state, but a proof by this method has not been forthcoming. For instance, in three dimensions the Fourier transform operator has an eigenvector that is a fiducial state (the one associated with the eigenvalue $i$ ), but this does not hold in general. In five dimensions a fiducial vector can be found among the degenerate eigenvectors of a particular $\mathbb{Z}_{3}$ subgroup of the normalizer of $\mathbb{Z}_{d} \times \mathbb{Z}_{d}$ in $S U(d)$, but there is no such subgroup at all in the normalizer for dimension seven. The group-theoretic structure of SIC-POVMs is exceedingly rich, however, and ongoing efforts to understand the full automorphism group of a SIC-POVM might yield insights into operators that yield fiducial states. We hope that by establishing the framework and providing motivating numerical results in this paper, a proof might be completed.

Finally, regardless of whether their existence can be proved rigorously, SIC-POVMs appear to exist in many dimensions. They might be of use in many areas of quantum information theory - signal ensembles, quasidistributions on discrete phase space, error-correcting codes, and quantum measurement, just to name a few. In addition, they appear to be connected to a number of interesting mathematical problems, including spherical codes and the putative existence of mutually unbiased bases.

Acknowledgements The authors gratefully acknowledge assistance and advice from H. Barnum, C. A. Fuchs, K. Manne, J. P. Paz, W. K. Wootters, and W. H. Zurek. This work was supported in part by Office of Naval 
Research Grant No. N00014-00-1-0578.

[1] E. Prugovečki, International Journal of Theoretical Phyics, 16, 321 (1977).

[2] P. Busch, International Journal of Theoretical Physics, 30, 1217 (1991).

[3] F. E. Schroek, Quantum Mechanics on Phase Space, (Kluwer, Dordrecht, the Netherlands, 1996).

[4] P. W. H. Lemmens and J. J. Seidel, "Equiangular Lines," Journal of Algebra 24, 494-512 (1973).

[5] P. Delsarte, J. M. Goethels, and J. J. Seidel, "Bounds for Systems of Lines and Jacobi Polynomials," Philips Research Reports 30, 91-105 (1975).

[6] H. König and N. Tomczak-Jaegermann, "Norms of Minimal Projections," arXiv:math.FA/9211211.

[7] S. G. Hoggar, "64 Lines from a Quaternionic Polytope," Geometriae Dedicata 69, 287-289.

[8] A. Koldobsky and H. König, "Aspects of the Isometric Theory of Banach Spaces," in Handbook of the Geometry of Banach Spaces, Vol. 1, edited by W. B. Johnson and J. Lindenstrauss, (North Holland, Dordrecht, 2001), pp. 899-939.

[9] T. Strohmer and R. Heath, "Grassmanian Frames with Applications to Coding and Communication," Appl. Comp. Harm. Anal 14, 257-275 (2003).

[10] H. König, "Cubature Formulas on Spheres," available online at http://analysis.math.uni-kiel.de/koenig/ preprints.html.

[11] B. Et-Taoui, "Equiangular Lines in $C^{r}$," Indagationes Mathematicae 11, 201-207 (2000).

[12] B. Et-Taoui, "Equiangular Lines in $C^{r}$ (Part II)," Indagationes Mathematicae 13, $483-486$ (2002).

[13] C. M. Caves, C. A. Fuchs, and R. Schack, "Unknown Quantum States: The Quantum de Finetti Representation," J. Math. Phys. 43, 4537-4559 (2002).

[14] C. A. Fuchs and M. Sasaki, "Squeezing Quantum Information through a Classical Channel: Measuring the 'Quantumness' of a Set of Quantum States," Quant. Info. Comp. 3, 377-404 (2003).

[15] C. A. Fuchs, "Quantum Mechanics as Quantum Information (and only a little more)," quant-ph/0205039, and private communication.

[16] G. M. D'Ariano, P. Perinotti, and M. F. Sacchi, "Informationally Complete Measurements and Groups Representation," arXiv:quant-ph/0310013.

[17] R. F. Werner, "All Teleportation and Dense Coding Schemes," Journal of Physics A 34, 7081-7094 (2001).

[18] J. J. Benedetto and M. Fickus, Advances in Computational Mathematics, 18, 357-385 (2003).

[19] A. Klappenecker and M. Rötteler. "Beyond Stabilizer Codes I: Nice Error Bases," IEEE Transactions on Information Theory IT-48, 2392-2395 (2002).

[20] A. Klappenecker and M. Rötteler. "Unitary error bases: Constructions, equivalence, and applications," Applied Algebra, Algebraic Algorithms and Error-Correcting Codes, Proceedings; 2643, 139-149 (2003).

[21] Klappenecker and Rötteler maintain an online catalog of nice error bases available at http://faculty.cs.tamu.edu/ klappi/ueb/ueb.html.

[22] E. Knill, "Non-binary Unitary Error Bases and Quantum Codes," LANL report LAUR-96-2717, available at arXiv: quant-ph/9608048.

[23] E. Knill, "Group Representations, Error Bases and Quantum Codes," LANL report LAUR-96-2807, available at arXiv: quant-ph/9608049.

[24] D. Han and D. R. Larson, "Frames, Bases, and Group Representations," Memoirs of the American Mathematical Society 147, 697 (2000).

[25] Available online at http://info.phys.unm.edu/papers/reports/sicpovm.html 